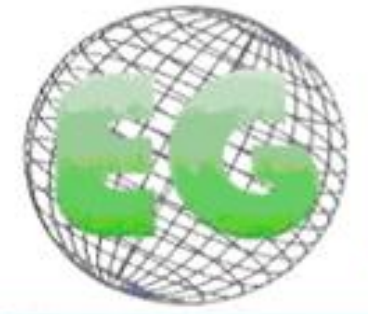

No 39

www.um.es/egloball

\title{
REVISIONES
}

\section{Evolución de la producción científica de historia de la enfermería en España entre1966 y 2013}

Evolution of scientific history production in Spanish nursing 1966-2013

\section{*Mínguez Moreno, Inmaculada **Siles González, José}

\author{
*Doctoranda del Departamento de Enfermería. Universidad de Alicante. Profesora asociada \\ Universidad de Zaragoza. E-mail: rodiles78@hotmail.com ${ }^{* *}$ Catedrático de la Universidad de
} Alicante. Facultad de Ciencias de la Salud. España.

Palabras clave: Historia de la enfermería; España; países anglosajones

Keywords: Nursing history; Spain; English-speaking countries

\section{RESUMEN}

Objetivos: Conocer la evolución y visibilidad internacional de la producción científica de historia de la enfermería en España en la segunda mitad del siglo XX hasta la actualidad. Conocer cuáles son las Universidades españolas que más producción científica tienen sobre la historia de la enfermería española durante el periodo de estudio. Comparar la producción científica sobre historia de la enfermería en países anglosajones y España.

Método: Se realizó un estudio de corte cualitativo a través de una revisión integrativa. Se utilizó como recurso secundario: Sciverse Scopus. Base de datos que incluye otras bases. Se incluyeron aquellos documentos que versaran sobre historia de la enfermería.

Resultados: La producción científica de historia de la enfermería en España no se muestra como uno de los campos de mayor interés para la investigación (10,80\%). La Universidad de Alicante se muestra como la Universidad Española con más producción en historia de la enfermería. Existe una clara diferencia en la producción científica sobre historia de la enfermería entre países anglosajones y España.

Conclusiones: España es el decimo país en producción científica de historia de la enfermería de un total de ciento cuatro incluidos en la base de datos. La producción científica española sobre historia de la enfermería sigue siendo modesta, comparada con los países anglosajones Existen sesgo sobre el estudio de la historia de la enfermería en una base de datos internacionales como es Sciverse Scopus. La no inclusión de revistas como Hiades (de dedicación exclusiva al estudio de la historia de enfermería) o la indexación parcial de bases de datos como Index (incluida sólo desde el año 2007) puede ser la causa 


\section{ABSTRACT}

Objectives: Know the evolution and the international visibility of the scientific production in nursing history in Spain, in the second half of XX century to the present. Know which Spanish Universities have more scientific production about the history of Spanish nursing, during the studied period. Compare the scientific production about English-speaking history nursing countries and Spain.

Methods: A qualitative studied was used through an integrative review. A secondary source was used: Sciverse Scoups which included other bases. All documents which were about nursing history were included.

Results: The scientific production about nursing history in Spain, it isn't showed as one of the most interesting fields for the investigation. (10,80\%). The University of Alicante it is shown as the Spanish University which has more nursing history production. There is an important difference in the scientific production between English-speaking countries and Spain.

Conclusions: Spain is the tenth country in scientific production of one hundred and four countries included in database. The Spanish scientific production continues being modest if it is compared with English speaking countries. The scientific Spanish production continues being modest if it is compared with English speaking countries. There is bias about Hiades (which have exclusive dedication to the nursing history) or the partial indexation of INDEX (only included since 2007) can be the cause.

\section{INTRODUCCIÓN}

La investigación es la base para el desarrollo de cualquier profesión. De ella surgen las mejoras aplicables posteriormente a la práctica. La producción científica se identifica en la actualidad como el cierre al proceso creador más allá de lo teórico y como manifestación práctica de los intereses de los profesionales ${ }^{(1)}$. En el caso de la enfermería, como profesión joven que es, el proceso de estudio de la producción científica es breve en términos históricos además de muy heterogénea ${ }^{(2)}$. Varios son los factores condicionantes de este retraso en el estudio y análisis de la producción científica. Apenas son los años 80 cuando comienza a percibirse un comienzo en la producción científica y cuando se encuentran los primeros estudios en lo que a enfermería en España se refiere.

Si nos centramos en el estudio de la historia de la enfermería, este se vuelve más caprichoso si cabe; ya que la conceptualización del estudio de la historia de lo cotidiano $^{(3)}$, como puede ser el trabajo enfermero, se sitúa a principios del XX con la Escuela de los Annales (1929). No es hasta ese momento en el que lo cotidiano se vuelve objeto de estudio. A esto hay que añadir que el estudio de la historia de enfermería en España se retrasa por las circunstancias históricas atravesadas en la segunda mitad del siglo ${ }^{(4)}$ que actuaron como asfixiante de la profesión y por lo tanto, enlentecieron más el proceso de estudio. Distintos autores han contribuido de forma extraordinaria al desarrollo y conocimiento de la historia de la enfermería en España en los últimos cuarenta años: Manuel Amezcual ${ }^{(5-7)}$ Juana Hernández Conesa $\left({ }^{8,9}\right)$, José Siles $^{(10-12)}$ Concha Germán ${ }^{(13,14)}$, Rosa Mํ- Alverdi, Carmen Domínguez Alcón ${ }^{(15)}$, Francisca Hernández Martin y Carmen Chamizo son algunos de los que han colaborado para conocer la historia de esta profesión en España.

La intención de este artículo es:

- Conocer la evolución y visibilidad internacional del estado de la producción científica de historia de la enfermería en España, en el universo: enfermería española, en la segunda mitad del siglo XX hasta la actualidad. 
- Conocer cuáles han sido las Universidades españolas que más han aportado a la historia de la enfermería en lo que a producción científica española se refiere en ese periodo de tiempo.

- Comparar la producción científica sobre historia de la enfermería en países anglosajones y España en ese periodo de tiempo.

\section{METODOLOGÍA}

La búsqueda se realizó el 10/01/2014. Fue de carácter temático, realizando una revisión integrativa ${ }^{(16)}$ que permitiera comprender de forma de completa el fenómeno pudiendo analizar, identificar y sintetizar los datos encontrados

En un primer momento se planteó el uso de bases de datos como Index o Scielo, pero se descartaron cuando se decidió incluir en el estudio un perfil tanto iberoamericano como anglosajón. Finalmente se decidió escoger una base de datos que siguiendo las recomendaciones de la Agencia Nacional de Acreditación y Evaluación de la Calidad y Acreditación (A.N.E.C.A), la define como una de las herramientas más potentes para este proceso, además de ser una herramienta con un perfil que homogeniza lo anglosajón con lo iberoamericano y lo europeo; revisando la idoneidad para este objetivo, finalmente Sciverse Scopus fue la elección.

La fuente utilizada nos permite consultar documentos desde 1945 hasta la actualidad y recoger más de 1500 divulgaciones. Es una base multidisciplinar, que no solo permite ver la producción enfermera que hay en revistas enfermeras, sino también publicaciones enfermeras en otros ámbitos. Además los documentos han sido revisados por pares y existe una referencia institucional (algo necesario para la consecución de uno de nuestros objetivos), si la fuente lo refleja.

Se utilizaron como palabras claves en inglés: "Spanish Nursing" y "Nursing history spain" para la consecución de los dos primeros objetivos. Para el tercer objetivo se utilizaron como palabras claves "Nursing history" acompañadas del país a estudio, estos son: E.E.U.U, Canada, Australia, Gran Bretaña y España. En un primer momento se dudó sobre si las palabras claves serian las más adecuadas pero una primera búsqueda dejó claro que esas palabras eran las que más ajustaban la búsqueda de los objetivos perseguidos, ya que eran las palabras clave que más artículos sobre cada uno de los temas de interés recuperaron. Los criterios de inclusión y exclusión fueron los mismos para todas las búsquedas.

Los criterios de inclusión fueron:

- Documentos escritos desde 1953, momento en el que se unifica el título de enfermera, matrona, y practicante, en España, hasta 2013.

- Para la búsqueda de "spanish nursing" los campos que se incluyeron fueron: medicina, enfermería, ciencias sociales, psicología, el motivo, que en cada uno de ellos se encontraron artículos que versaban sobre la enfermería española.

- Para la búsqueda de "spanish nursing history" y el estudio de la historia de la enfermería de los distintos países anglosajones se incluyeron los campos de medicina, enfermería, ciencias sociales. Campos en los que quedaba recogida 
toda la información que sobre historia de la enfermería española o de los países a estudio quedaba recogida.

- No se limitó el idioma.

Los criterios de exclusión fueron:

- Aquellos artículos rescatados que no fueran sobre historia de la enfermería

\section{Limitaciones}

- Entre los límites que se pueden establecer en la base de datos Sciverse, se encuentra la temática a buscar. Se decidió no limitar la temática a "nursing" ya que los documentos anteriores a 1980 se perdían, teniendo una ligazón realmente enfermera. Eso a su vez arrojó ciertos documentos, en concreto cuatro que hubo que desestimar después de revisar de una manera artesana la búsqueda completa.

- La base de datos no recoge datos previos a 1966, y este estudio pretendía conocer la situación desde 1953 hasta 2013, teniendo al final que ser reducido por causas evidentes.

- Tampoco se limitó el idioma, ya que al hacerlo se perdían artículos, por dos causas principalmente :

- Existen documentos escritos en otros idiomas, como en checo, que hablan sobre la guerra civil española y enfermería.

- Existen artículos de autores españoles escritos en inglés.

- La no inclusión de revistas como Hiades o la indexación únicamente de Index desde el año 2006, hace que en este estudio no aparezca información muy valiosa sobre historia de la enfermería en España, dando una visión sesgada de la situación y del estudio de la historia de la enfermería española.

\section{RESULTADOS}

Para poder conocer qué cantidad de producción científica ocupa la historia de la enfermería española, se hace necesario conocer cuánta es la producción científica del universo enfermería española, con el fin de establecer qué porción del total se le asigna al estudio de la historia. Sciverse Scopus nos muestra documentos sobre "spanish nursing" desde 1965, mostrando un total de ochocientos cinco documentos. El primer registro es un artículo del año 1966. 
FIG. 1.-Evolución de la producción científica española 1966-2012(extraído de Sciverse Scopus)

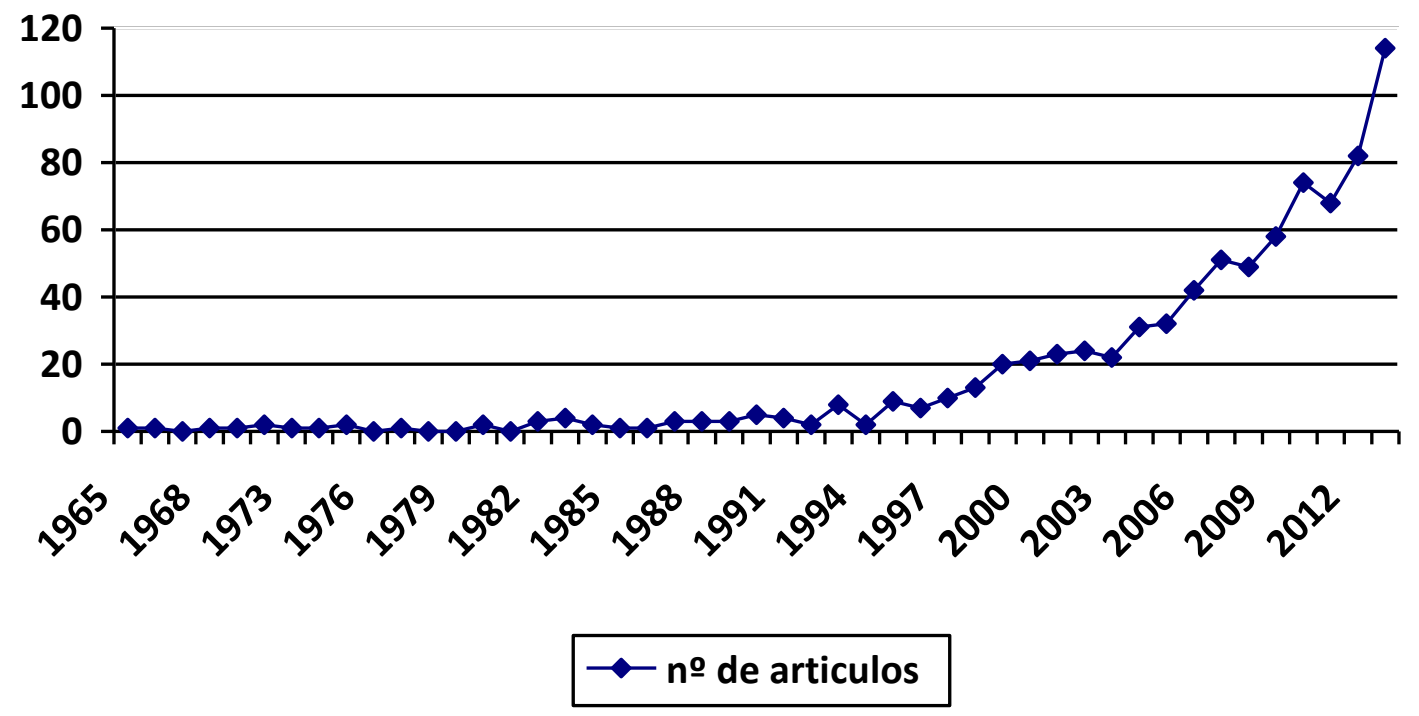

Respecto a la búsqueda sobre "nursing history spain", se obtuvieron ciento treinta y un artículos. De esos ciento treinta y un artículos, cuarenta y dos fueron descartados por no versar sobre historia de la enfermería en España, causa sobre todo de no limitar solo la búsqueda a la temática: enfermería. Finalmente se encontraron ochenta y nueve artículos (Anexo1).

Sciverse Scopus, solo nos arroja información desde el año 1966, con un único artículo, sobre la guerra civil española, de origen checo $^{(17)}$. No sería hasta el año 1970 en el que se encuentra el siguiente registro con únicamente dos artículos, uno el año 1971 y 1974 y cuatro en 1975. En los años 1972, 1973, 1976, 19771978 y 1979 son años en los que no se encuentra ningún registro. No es hasta la llegada de la década de los ochenta donde se recupera la producción científica. 1980 es el momento en el se encuentran datos de nuevo, con un único artículo, dos artículos en 1981, uno en 1982, dos en 1983, cuatro en 1984 y 1985, cuatro en 1986 para bajar a uno en 1987 y tres en 1988. En el año 1989 no se recoge ningún registro. Durante la década de los noventa solo se encuentran registros pertenecientes a los años 1992, 199319941995 y 1999, con uno, dos, dos, cinco y un artículos, respectivamente. Desde el año 2000 al año 2006, los registros arrojan una producción de artículo por año, exceptuando el año 2004, donde hay dos, y el año 2001 donde no hay ningún registro. Desde 2007, se produce un incremento claro, comenzado ese año con tres documentos, cuatro en 2008 y 2009, tres en 2010, solo uno en 2011 y doce y trece respectivamente en 2012 y 2013. 
FIG. 2.-Evolución de la producción científica historia de la enfermería España 19662013(extraído de Sciverse Scopus)

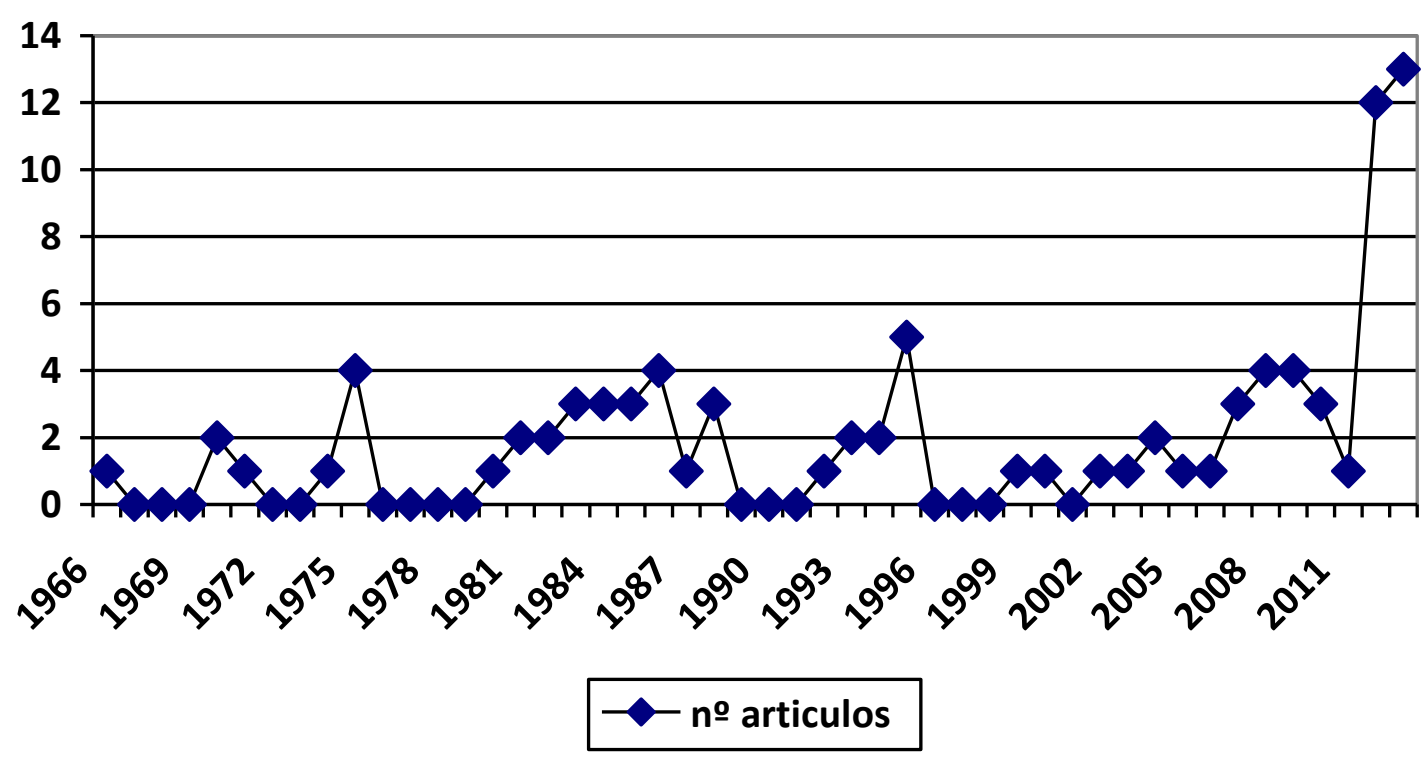

En cuanto a las aportaciones que desde las Instituciones Universitarias españolas, sobre historia de la enfermería se han realizado, Sciverse Scopus muestra la Universidad de Alicante como la Institución Universitaria Española como la que mas aportaciones ha hecho en producción científica de historia de la enfermería. Destacan otras universidades como la Autónoma de Barcelona, Universidad de Murcia.

FIG. 3.-Aportaciones de las Universidades Españolas a la producción científica de historia de la enfermería España (extraído de Sciverse Scopus)

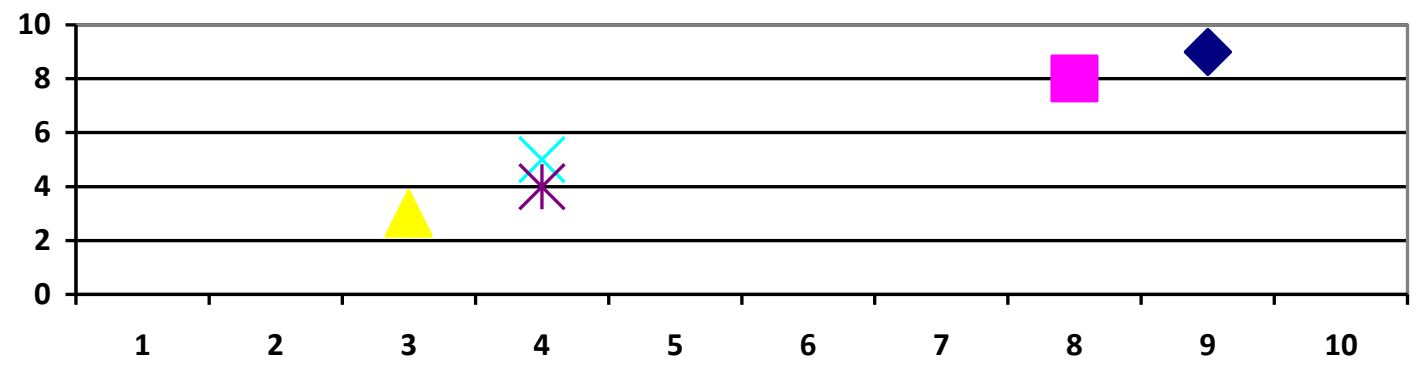

\begin{tabular}{|ll|}
\hline- Universidad de alicante & - - Universidad de Barcelona - Universidad de Murcia \\
- Universidad Autonoma & $\rightarrow-$ Universidad de Granada \\
\hline
\end{tabular}

Con respecto al tercer y último objetivo, cuando hablamos de países anglosajones, incluimos: Canadá, E.E.U.U, Australia y Gran Bretaña. Al realizar la comparativa de la producción científica entre países anglosajones y España, los datos son claros, los cuatro países anglosajones ocupan los cuatro primeros puestos de la lista, siendo el primero E.E.U.U con tres mil doscientos cincuenta y ocho, el segundo Gran Bretaña con ochocientos cincuenta, el tercero Australia con trescientos ochenta y seis, y el cuarto Canadá con trescientos setenta y tres España se sitúa en un décimo puesto, con un total de ochenta países. Teniendo por encima países como Brasil, Alemania Francia, Japón o Países Bajos. 
FIG. 4.-Comparativa a la producción científica de historia de la enfermería entre países anglosajones y España (extraído de Sciverse Scopus)

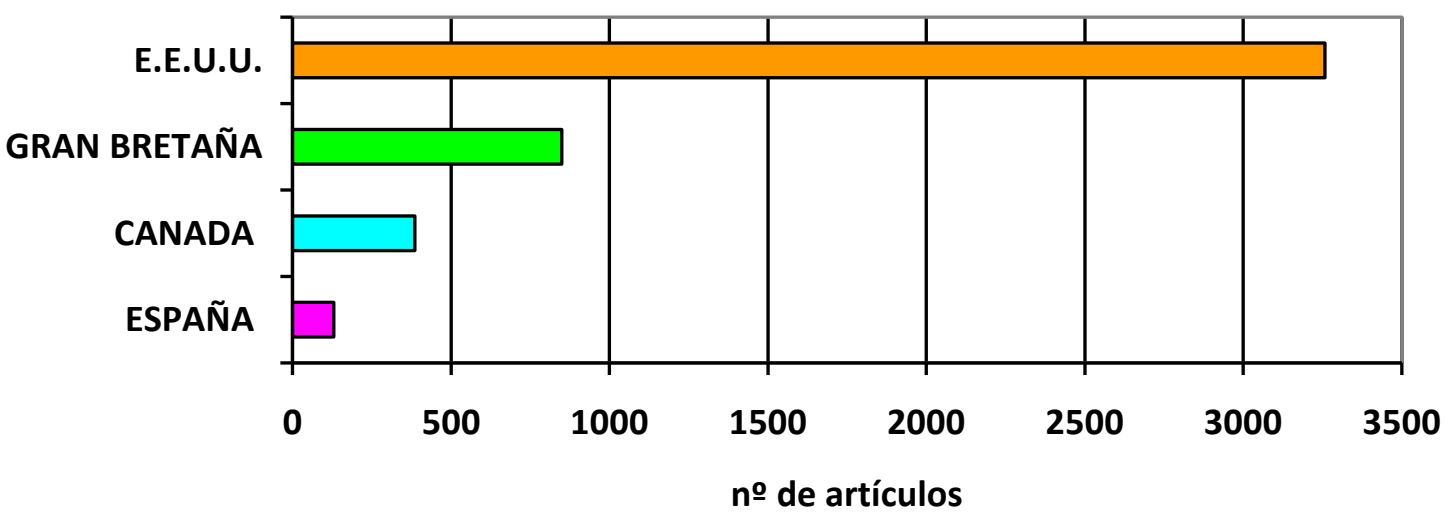

$\square$ ESPAÑA $\square$ CANADA $\square$ GRAN BRETAÑA $\square$ E.E.U.U.

\section{DISCUSIÓN}

El universo "enfermería" existente en Sciverse Scopus, es de ochocientos cinco documentos. Este estudio pretendía estudiar el periodo de tiempo comprendido entre 1953 y 2013. Sciverse Scopus solo recoge documentos enfermeros desde el año 1966. Cierto es que en otros estudios muestran ${ }^{(18)}$ una tímida existencia de producción científica enfermera en España en los años previos al años 1966, caracterizada por la unión a los colegios y al colectivo médico, que en este estudio no quedan reflejados a causa de la no existencia de registros en esas fecha en la base ${ }^{(18)(13)}$. Según distintos estudios $1977^{(19)(18)}$, con la entrada a la Universidad de la profesión, es el momento en el que se produce un aumento en las publicaciones periódicas. En esta investigación esto queda de manifiesto pasando de los nueve artículos de la década de los 70 a los veinte en la de los ochenta. A pesar de ese aumento, aún existe cierta dificultad para definir "el ser" de la profesión, cosa que se manifiesta a través de las aún discretas cifras de producción. Sin unas funciones claras, la investigación queda en cierta manera desplazada ${ }^{(20,21)}$. Autores como Camaño y Amezcua ${ }^{(5,22)}$ no solo achacan este cambio a la llegada a la Universidad sino también a la aparición del código deontológico, que produjo importantes cambios en la conceptualización de la profesión tanto de los profesionales, como de los usuarios.

La década de los 90 se muestra en este estudio con una curiosa bajada en la producción con respecto a los años ochenta. Esto choca con la creación de un Grupo de Trabajo de Enfermería con dinero del Fondo de Investigación Sanitaria (PIS), que entre otras actividades, establece pautas para que la investigación en enfermería se produzca.

Comienza a haber publicaciones de cómo investigar, con el fin de crear una cultura investigadora y científica, y por último surge la revista Index de Enfermería, que nace con una clara intención: "ocupar un lugar en el campo incipiente de la documentación enfermera. Su ámbito de interés abarca el del conocimiento de los elementos prácticos del ciclo de producción bibliográfica, entendido en su sentido amplio, que va desde el acceso a la información publicada, hasta las formas de presentación y publicación de trabajos» ${ }^{(23)}$

Poniendo esto los puntales para el posterior y definitivo desarrollo de la producción. 
Esta dicotomía que se plantea en la década de los noventa con los datos encontrados y el gran desarrollo que supuso la aparición de Index, puede ser atribuible a ciertas limitaciones de Sciverce Scopus:

- Por un lado, Index aparece indexada en la base únicamente desde el año 2006, con lo que todos los registros existentes desde su creación hasta este año no se muestran.

- Y por otro lado causa de la limitación de la base es la revista Hiades, dedicada en exclusiva desde el año 1993 al estudio de la historia de enfermería española, que tampoco está incluida. En ella se aborda de una forma completa la investigación histórica incluyendo: Monografías, fichas, eventos sobre historia, investigación en las aulas. Esto hace que muchísima información relevante para el estudio de la historia de la enfermería se pierda.

Todo lo sucedido en las décadas previas, permite que se inicie de una forma clara y progresiva la producción científica en España llegado el año 2000, momento en el que la producción científica enfermera Española además de todo los sucedido se ve influida por la llegada de los grados y los postgrados en Europa ${ }^{(22)}$. Pero no es hasta 2007 el momento en el que la enfermería se muestre como una disciplina que va madurando mostrando visos de poder formar parte de lo que Amezcua denomina como Gran ciencia. Durante el proceso de investigación quedó claro que el uso o no de documentos escritos antes o después de la puesta en marcha en España del plan Bolonia, puede cambiar mucho los datos, ya que aunque algunos estudios previos ya predecían el impacto que tendría ésta en la producción $\left({ }^{18)}\right.$, las cifras y la visibilidad internacional son distintas ${ }^{(24)(25)}$

En el año 2007, los indicadores bibliométricos del grupo de estudios documentales de la Fundación Index (G.E.D) dan muestras incluso de un perfil investigador concreto de la enfermería española: Con un aumento en la demanda de conocimiento enfermero y donde la enfermería española consume producción científica. De forma resumida Gálvez del Toro lo resume como: "encontrar lo que necesita para dar respuesta a sus problemas, que eso sea fácil, a ser posible en su idioma y a texto completo. Eso es lo que la gente quiere lo manifiesta con citas y referencias. Es lo que certifican los indicadores bibliométricos" (26)

Del total de los documentos encontrados, ochocientos cinco, solo ochenta y siete versan sobre historia de la enfermería en España, lo que representa un 10,80\% del total. Si hacemos un cómputo por décadas de la producción sobre historia de la enfermería desde el año 1966 hasta el año 2013, la evolución y distribución de la producción científica es progresiva, exceptuando la década de los noventa donde se produce una bajada de la producción, para en la primera década del siglo veintiuno proseguir con ese aumento.

Es llamativamente importante la producción científica en historia de la enfermería en España en los últimos tres años, veinticuatro artículos, comparable al volumen de producción de décadas como los ochenta o la primera década del siglo veintiuno. Este desarrollo estuvo impulsado por autores que desde al año 1953 hasta 1984 actúan como impulso creador de estudios sobre la materia ${ }^{(6,18)}$. En este estudio quedan diferenciados dos periodos claros: desde 1953 a 1977 y desde 1977 hasta la actualidad. Con la unificación del título en 1953 surgen distintos escritos sobre historia como es el manual: Historia de la Profesión (practicante-enfermera-Matrona) ${ }^{(27)}$ A partir de 1977, con la entrada en la Universidad, aparecen escritos sobre historia de 
la enfermería como el de Alberdi Castells y col, que apoyan el cambio producido desde una perspectiva histórica y ya en los años ochenta, surgen algunos artículos $^{(6)(25)}$. Clara y de manifiesto queda tanto en nuestra investigación como en otras, un antes y un después en el desarrollo de la producción científica en historia de la enfermería, en cuanto a forma y contenido. Todo ello impulsó congresos sobre la historia de la enfermería en los años noventa mostrándose como la vía de expresión en los primeros años de desarrollo de la producción científica en historia. Es cierto que existe cierta dificultad a la hora de agrupar por materias los documentos, pero hay estudios que coinciden con lo encontrado en esta investigación en que la historia de la enfermería no se sitúa como una de las materias más estudiadas en España ${ }^{(6)}$. Existen estudios ${ }^{(7,23,26)}$ que detectan un incremento del consumo de la literatura del espacio iberoamericano, esta conducta es compatible con la madurez y muestra un aumento del en ese ámbito. ${ }^{(28)}$

Si hablamos de la producción científica sobre historia de la enfermería en países anglosajones y España en ese periodo de tiempo, el estudio deja de manifiesto que los países anglosajones son, con diferencia, los que más producción científica tienen sobre historia de la enfermería. España se nuestra en un discreto décimo puesto (dentro de una lista con un total de ciento cuatro países), lejos aún de los países anglosajones.

Distintos autores ${ }^{(29,30)}$ achacan esta invisibilidad a lo inadecuado del uso de bases de datos internacionales como SCl, SSCi,.etc., ya que desfavorece y desmerece la enfermería y más si cabe la española. Consideran que se produce una doble marginación ${ }^{(25)}$, por un lado el idioma y por otro el que estos medios tienen hacia determinadas áreas. Cuando se consultan bases como Medline o la Web of Science del ISI, se confirma, causa de los pocos datos incluidos en esas bases sobre enfermería española.

En cambio Sciverse Scopus, siendo una base de datos internacional y multidisciplinaria que encierra una amplia cobertura y donde más de la mitad del contenido es Europeo, Latinoaméricano y la región Asia-Pacífico. Con casi 18000 títulos (un $47 \%$ son revistas europeas), más de 5.000 editores internacionales de ciencias sociales, medicina, humanidades, etc. De hecho el idioma no se limitó tras observar que no solo desde el territorio español se producía el estudio de la enfermería española y no solamente se escribe en castellano desde España.

Con respecto a las aportaciones de las instituciones, no se encontraron estudios específicos sobre las aportaciones a la producción científica del estudio de la historia de la enfermería en España por parte de las instituciones. Estos señalan que la producción científica encontrada es mayor por parte de instituciones hospitalarias, pero cierto es que son estudios de los años 90 , y ha sido después cuando el desarrollo en investigación ha sido mayor. Estudios posteriores hablan de la dificultad para la atribución de la autoría tanto a nivel individual como de instituciones por la heterogeneidad en la forma de recoger los datos ${ }^{(31,32)}$.

En este estudio las instituciones que más aportación han hecho a la producción sobre historia de la enfermería son Universidades; siendo la Universidad de Alicante la que más aportaciones ha hecho. Este dato no coincide con otros estudios donde se afirma que es el ámbito hospitalario el que más aporta. No es de extrañar ya que todos los artículos consultados estudiaban la producción científica en términos generales y no sobre historia de la enfermería. No se encontró ningún artículo que revisara solo la producción histórica de la enfermería ${ }^{(32)}$. 


\section{CONCLUSIONES}

$>$ El estado de la producción científica de historia de la enfermería en España, en el universo: enfermería española, en la segunda mitad del siglo XX hasta la actualidad, a pesar de la las aportaciones en los últimos años, no se muestra como uno de los campos de mayor interés para la investigación $(10,80 \%)$ y por tanto se hace necesario su estudio.

> La Universidad de Alicante se muestra como la Universidad con más producción en historia de la enfermería, de las instituciones recogidas en la base de datos.

> Los países anglosajones muestran una clara diferencia en la producción científica sobre historia de la enfermería, con respecto a España, ocupando los cuatro primeros puestos de la lista y España el décimo.

$>$ Cabe destacar que aunque la producción científica española sobre historia de la enfermería sigue siendo modesta, comparada con los países anglosajones, España ocupa el décimo puesto de un total de ciento cuatro países incluidos en la base de datos utilizada para el estudio.

> Sigue existiendo cierto sesgo sobre el estudio de la historia de la enfermería en base de datos internacionales como es Sciverse Scopus, causa de la no inclusión de revistas como Hiades( de dedicación exclusiva al estudio de la historia de enfermería) o la indexación de bases de datos como Index( incluida sólo desde el año 2007).

> La visibilidad de la producción científica enfermería en España, en una base de datos internacional como es Sciverse Scopus, parece estar cambiando tras la llegada del Plan Bolonia a las Universidades españolas.

> Estudios posteriores comparativos con bases de datos nacionales pueden mostrar si estos datos muestran de forma real a nivel internacional, el estado de la producción de la enfermería española en general, y de la historia de la enfermería

\section{REFERENCIAS}

(1) Jiménez Contreras E. Las revistas científicas: el centro y la periferia. Rev Esp Doc Cient 1992;15(2):174-182.

(2) Jiménez Contreras, E. Las revistas científicas: el centro y la periferia. Esp Doc Cient 1992;15(2):174-182.

(3) Legout, S. The "Annales de I'Institut Pasteur", 1887-2007: a glimpse into history. Res Microbiol 2008;159(1):23-26.

(4) Minguez, I. Siles, J. Crisis de identidad enfermera: Origen y... ¿superación a través de la práctica reflexiva en el entorno comunitario? RIDEC 2010;3(2):5-8.

(5) Amezcua, M. Galvez del Toro, A. Heierle Valero, C. Poyatos Huertas,.E. La pequeña ciencia.Producción, repercusión y trasmision del conociemiento.La enfermería del area lingüistica del español y del portugues. 2003 2003:82.

(6) Amezcua, M. Los estudios y los estudiosos de los cuidados en España. Lo histórico como elemento de identidad. Index de Enfermería [Index Enferm] (edición digital) 1993;4-5.:http://www.index-f.com/index-enfermeria/4-5/1624.php.

(7) Galvez Toro, A, Amezcua, M. Salido Moreno, M ${ }^{a}$ paz. Hueso Montoro, C. Repercusión e impacto de las revistas de enfermería del espacio iberoamericano. Index Enferm 2006 2007;Año XV(57):83-86.

(8) Conesa H, María J. Historia de la Enfermería. Un Análisis Histórico De Los Cuidados De Enfermería 1995. 
(9) Conesa, JMH. Madrid, MJT. Perán, AN. Relation between the politico-educational ideology of the Franco regime and contents of compulsory subjects such as professional moral and religion in nursing studies. Revista de Investigacion Educativa 2013;31(1):199-221.

(10) Siles, J. Historia de la enfermería. Alicante: Aguaclara; 1999.

(11) Siles, J. Antropología y enfermería. La necesaria simbiosis entre dos disciplinas para vertebrar culturalmente la teoría y la praxis de los cuidados. Index Enferm 2003;43:28-32.

(12) Siles González, J. Pasado, presente y futuro de la Enfermería en España. Perspectiva Histórica Y Epistemológica 1996.

(13) Germán, C. Historia de la institución de la enfermería universitaria. Análisis con una perpectiva de género. Zaragoza: Universidad de zaragoza; 2006.

(14) Suárez Prieto, A. Germán Bes, C. The baker's wife: a nursing predecessor. Rev Enferm 1986;9(94):25-27.

(15) Chamizo Vega C. Historia, sincronicidad y memoria en la enfermería española. Index De Enfermería 2004;13(47):35-38.

(16) Souza, MT. Silva ,MD. Carvalho, R. Revisão integrativa: o que é e como fazer. Rev Einstein 2010;8(1 Pt 1):102-6.

(17) Anniversary of the Spanish civil war. Cesk Zdrav 1966;14(10):505-510.

(18) Jiménez Hernádez, J. Ayuso Garcia, M. Murrillo Murillo, R. Guillen Rios, .J. Evolución de las publicaciones periódicas españolas de enfermería. index Enfem(Gran) 2007.Primavera;56:73-78.

(19)Gálvez Toro, A. Producir es existir. El conocimiento como gestor del ser. ENE 2008; Revista de enfermería(2):19-20.

(20) Montesinos, A. Investigación y enfermería. Rev Rol Enferm 1984;67:76-79.8.

(21) Mora Temprano, M. Investigación, herramienta que forja una profesión. Rev Rol Enferm 1986;100:40-44.

(22) Camaño, P. The state of the nursing science in Spain. . 2002; Available at: http://www.scielo.br/scielo.php?script=sci arttext\&pid=S0104-

$11692002000200014 \&$ Ing=pt. Accessed [access 2013 Jul 02]; 10(2):214-20, Rev Latino-am Enfermagem [online].

(23) Galvez Toro, A. Poyatos Huertas, E. index Enfem(Gran) 1998 primavera-verano; VII(20-21)11-15.

(24) Gálvez Toro, A. López Medina, IM. Sánchez Criado, V. Poyatos Huertas, E. Evaluación de la Actividad Científica de la Enfermería Española. Impacto y aislamiento en el año 2000. 2001; 34. Index de Enfermería [Index Enferm] (edición digital) Disponible en <http://www.index-f.com/indexenfermeria/34revista/34 articulo 54-64.php>.

(25) Sobrido Prieto, M. Sobrido Prieto, N. González Gutián, C. Pichel Guerrero, MJ. García Sánchez,MM. Prieto Díaz, A. Revistas españolas de enfermería en bases de datos nacionales e internacionales. index Enfem(Gran) 2005 primavera-verano;Año XIV(48-49): 74-75.

(26) Gálvez Toro A. La legitimidad del valor de la producción científica enfermera. Index Enfem(Gran) 2007;XVI(57):7-8.

(27) Álvarez Sierra y Manchón, Murga Serret, J. Historia de la profesión (enfermerapracticante-matrona). . Academia Murga Madrid 1995.

(28) Sanfeliu V, Elias A, Figueras, D. Verdura, T. Gelabert, M. Piqué, JL et al. 15 years later. Rev Enferm 1993;16(178):12-17.

(29)López Piñero, J. Terrada, M. Los indicadores bibliométricos y la evaluación de la actividad médico científica. (II). La comunicación científica en las distintas áreas de las ciencias médicas. Medicina Clínica; 98; 101-106. 
(30) López Piñero, J. Terrada, ML. Los indicadores bibliométricos y la evaluación de la actividad médico científica. (I). Usos y abusos de la bibliometría. Medicina Clínica 1992;98; 64-68.

(31) Salido Moreno, M. Gálvez Toro, A. Ética y Enfermería. El estado de la cuestión en términos de producción científica. Ética de los cuidados.2008; Cuidados, 1 (1).

(32) Serrano Gallardo, P. Giménez Maroto, A. Arroyo Gordo, Mํ. Análisis de la producción científica publicada en la revista Metas de Enfermería. Index De Enfermería 2005;XIV(48-49): 78-82. 


\section{ANEXO 1}

\section{(Artículos incluidos en esta investigación)}

(1) Abad FEM. History of cures in moist enviroment (CME). The "spanish method" of wound war treatment and the swedish-norwegian hospital in alcoy. Gerokomos 2013;24(1):32-35.

(2) Bernabeu-Mestre J, Carrillo-García C, Galiana-Sánchez ME, GarcíaParamio P, Trescastro-López EM. Gender and profession in the historical development of community nursing in Spain. Enfermeria Clinica 2013;23(6):284-289.

(3) Blasco RM. [Thirty-five years later]. Revista de enfermería (Barcelona, Spain) 2013;36(6):52-53.

(4) Conesa JMH, Madrid MJT, Perán AN. Relation between the politicoeducational ideology of the franco regime and contents of compulsory subjects such as professional moral and religion in nursing studies. Revista de Investigacion Educativa 2013;31(1):199-221.

(5) Eseverri Chaverri C. [Spanish nursing, 1977-1978]. Revista de enfermería (Barcelona, Spain) 2013;36(4):31-32.

(6) García García I, Gozalbes Cravioto E. Rise and development of the history of nursing in Spain. Enfermeria Global 2013;12(2):304-314.

(7) Heierle Valero C. [Life history, accompanied by ROL]. Revista de enfermería (Barcelona, Spain) 2013;36(5):46-47.

(8) Hernández Conesa JM, López GS. The formation of the red cross nursesladies during the spanish civil war (1936-1939). Index de Enfermeria 2013;22(3).

(9) León Molina J. Nursing texts printed in spain during the sixteenth and seventeenth centuries available in digital libraries. Enfermeria Global 2013;12(4):280-287.

(10) Losa Iglesias ME, Becerro de Bengoa Vallejo R. History of bioethics in professional nursing education: A Spanish view. Acta Bioethica 2013;19(2):293-297.

(11) Miró-Bonet M, Bover-Bover A, Moreno-Mulet C, Miró-Bonet R, ZafortezaLallemand C. Genealogy as a critical toolbox: Deconstructing the professional identity of nurses. J Adv Nurs 2013.

(12) Romero-Collado A, Homs-Romero E, Zabaleta-del-Olmo E, Juvinya-Canal D. Nurse prescribing in primary care in Spain: Legal framework, historical characteristics and relationship to perceived professional identity. J Nurs Manag 2013.

(13) [ROL's 30th anniversary. We dedicate it to you, Néstor]. Revista de enfermería (Barcelona, Spain) 2012;35(7-8):6-7.

(14) Arroyo Gordo P. [A glance at the 35 years of evolution of nursing in 
Spain]. Revista de enfermería (Barcelona, Spain) 2012;35(7-8):17-18.

(15) García-García I, Cravioto EG. Research of nursing and history of nursing in Spain. Index de Enfermeria 2012;21(1-2).

(16) González Jurado M. [35 years of health excellence]. Revista de enfermería (Barcelona, Spain) 2012;35(7-8):38-39.

(17) Hernández Conesa JM, Cayuela Fuentes PS, Beneit Montesinos JV, González Jurado M. Spanish nurses's credentialing in the 20th century. Int Nurs Rev 2012;59(2):175-180.

(18) Miró M, Gastaldo D, Nelson S, Gallego G. Spanish nursing under Franco: Reinvention, modernization and repression (1956-1976). Nurs Inq 2012;19(3):270-280.

(19) Peya Gascóns M. [On the 35th anniversary of Revista ROL de Enfermería]. Revista de enfermería (Barcelona, Spain) 2012;35(7-8):50-52.

(20) Ribot Puig J. [Revista ROL is 35 years old]. Revista de enfermería (Barcelona, Spain) 2012;35(9):53-57.

(21) Ruiz Galiano P. [35th anniversary of the publication Revista ROL de Enfermería]. Revista de enfermería (Barcelona, Spain) 2012;35(7-8):42-44.

(22) Santos TC, Gomes ML, Oliveira AB, Almeida Filho AJ. [The Vargas Dictatorship in Brazil (1937-1945) and the Primer Franquismo in Spain (19391945): power and counter-power of nurses]. Rev Bras Enferm 2012;65(2):347352.

(23) Siles González J, Solano Ruiz MC. The convergence process in European Higher Education and its historical cultural impact on Spanish clinical nursing training. Nurse Educ Today 2012;32(8):887-891.

(24) Telford JC, Long TL. Gendered spaces, gendered pages: Union women in civil war nurse narratives. Medical Humanities 2012;38(2):97-105.

(25) do Prado ML, Medina-Moya JL, Martínez-Riera JR. Nursing education knowledge production in Spain and Brazil: An integrative review. Texto e Contexto Enfermagem 2011;20(3):407-415.

(26) Pérez-Aguado MC, Brugues E, de Leiva-Perez A, de Leiva A. Medicine and nursing in the Spanish Civil War: women who served in the health services of the International Brigades (1936-1939). Vesalius : acta internationales historiae medicinae 2010;Suppl:29-33.

(27) Santos TCF, de Alencar Barreira I, de Almeida Filho AJ, de Oliveira AB. The franco and vargas dictatorships: Implications of the consecration of maternity for nursing. Texto e Contexto Enfermagem 2010;19(2):317-324.

(28) Tuells J, Penadés EM. Women before the 1918 influenza: Accreditation of diseases. Vacunas 2010;11(1):33-36.

(29) Spanish nurse leader receives nursing's top international award. Int Nurs Rev 2009;56(2):150-153. 
(30) Boatella J. The first ready foods meant for maternal nursing registered in Spain (1919-1935). Actividad Dietetica 2009;13(4):173-177.

(31) Norrie C, Muller J, Goodson I, Hernandez F. Restructuring nurses' worklives and knowledge: Case studies from England and Spain. Int Nurs Rev 2009;56(1):81-87.

(32) Zabalegui A, Cabrera E. New nursing education structure in Spain. Nurse Educ Today 2009;29(5):500-504.

(33) Fernández Lamelas MA, Alvarez Rodríguez T, Ramiro Fernández JM, Martínez de Santiago $S$. The privacy respect and the professional secret in nursing. Cuadernos de bioética : revista oficial de la Asociación Española de Bioética y Ética Médica 2008;19(65):59-66.

(34) Linares Abad M, Álvarez Nieto C, Moral Gutiérrez I. Medical discuss about midwives on the half of century XX. Index de Enfermeria 2008;17(4).

(35) Manrique Sáez MP, Ortega Larrea S, Yanguas Jiménez P. The leeches, a worm in the history of health. Index de Enfermeria 2008;17(4).

(36) Miró-Bonet M, Gastaldo D, Gallego-Caminero G. Why are we the way we are? Discourses and power relations in nurses' professional identity in Spain (1956-1976). Enfermeria Clinica 2008;18(1):26-34.

(37) Coll M, Besora I, Icart T, Vall AF, Manito I, Ondiviela A, et al. Nursing care according to Virginia Henderson in the at home care field. Revista de enfermería (Barcelona, Spain) 2007;30(3):53-56.

(38) de la Torre Aboki J. The formation of the Spanish Rheumatology Nursing Society. Musculoskeletal care 2007;5(1):1-3.

(39) Gallego Caminero G, Bosch Cladera MP, Miró Bonet M, Sánchez-Cuenca López P, Zaforteza Lallemand C. Nursing treatments in Majorca. Revista de enfermería (Barcelona, Spain) 2007;30(6):24-32.

(40) González Canalejo C. Care and well-being: Female healthcare work in response to the «social question»(1857-1936). Dynamis 2007;27:211-235.

(41) Jiménez Rodríguez I. Syphilis in the XVII century, treatment in a hospital in Toledo. Revista de enfermería (Barcelona, Spain) 2006;29(11):46-53.

(42) Vázquez de Quevedo F. Instituto de Terapéutica Operatoria (1880-1939). Instituto Rubio y Gali, Instituto Moncloa. Contribution to medical specialities and nursing in Spain. Anales de la Real Academia Nacional de Medicina. 2005;122(3):411-426; discussion 426-430.

(43) Atance Martínez JC, Yusta Izquierdo A, Grupeli Gardel BE. Costs study in Alzheimer's disease. Rev Clin Esp 2004;204(2):64-69.

(44) Calvo JJ. Geriatric nursing. An option for the present and future. Revista Espanola de Geriatria y Gerontologia 2004;39(SPEC. ISS. 4):1-2.

(45) Soto A, Tofé S, León M, García-Luna PP. The organizational and clinical situation of clinical nutrition in Spanish hospitals: 1995-2001. Endocrinologia y 
Nutricion 2003;50(1):8-13.

(46) Zabalegui A. Nursing education in Spain - Past, present and future. Nurse Educ Today 2002;22(4):311-318.

(47) Anía Lafuente BJ, Suárez Almenara JL, Fernández-Burriel Tercero M, Guerra Hernández L, Betancort Mastrángelo $C$. Thyroid function in the aged admitted to a nursing home. Anales de Medicina Interna 2000;17(1):5-8.

(48) Jiménez Herrero F. Old people care and geriatrics teaching: Evolution in Galicia (Spain). Geriatrika 1999;15(6):33-40.

(49) Fargues García I, Tey Freixa R. History of nursing. "Rules from 1417". Hospital of Santa Creu of Barcelona. Rev Enferm 1995;18(200):47-53.

(50) Fargues García I, Tey i Freixa R. History of nursing. 16. and 17. centuries. Rev Enferm 1995;18(203-204):25-29.

(51) Pacheco Borrella G, Vila Blasco B. Evolution of psychiatric nursing in Andalusia. Rev Enferm 1995;18(198):37-40.

(52) Patai F. Heroines of the good fight. Testimonies of U.S. volunteer nurses in the Spanish Civil War, 1936-1939. Nursing history review : official journal of the American Association for the History of Nursing 1995;3:79-104.

(53) Wall BM. Courage to care. The Sisters of the Holy Cross in the SpanishAmerican War. Nursing history review : official journal of the American Association for the History of Nursing 1995;3:55-77.

(54) Campos Pérez J, Martínez Molina A, Gracia Pérez J. Nursing in the 16th and 17th centuries. Rev Enferm 1994;17(193).

(55) García Barrios S, Calvo Charro E. Florence Nightingale and nursing education. Rev Enferm 1994;17(194):41-43.

(56) The commitment of ROL, 15 years with, for and from Spanish nursing. Rev Enferm 1993;16(178):9.

(57) Sanfeliu V, Elias A, Figueras D, Verdura T, Gelabert M, Piqué JL, et al. 15 years later. Rev Enferm 1993;16(178):12-17.

(58) Velandia AL. The Spanish influence in Colombian nursing. Revista LatinoAmericano de Enfermagem 1993;1(2):129-150.

(59) Hernando Aizpúrua J, Iturrioz Rosell P, Torán Monserrat P, Arratibel Arrondo I, Arregui López A, Merino Hernando M. The health problems and needs of chronic home-bound patients. Atencion Primaria 1992;9(3):133-136.

(60) Alvarez-Dardet C, Gascón E, Alfonso M, Almero A. The origins of public health nursing. Gac Sanit 1988;2(9):290-293.

(61) Binder L, Nelson B, Smith D, Glass B, Haynes J, Wainscott M.

Development, implementation, and evaluation of a medical Spanish curriculum for an emergency medicine residency program. J Emerg Med 1988;6(5):439441. 
(62) Blasco RM. 10 years with a positive balance for nursing. Rev Enferm 1988;11(117):26-27.

(63) Celebration of the 100th issue of ROL gives credit to our nursing performance. Rev Enferm 1987;10(101):58-65.

(64) Guilera E. Development of nursing from the viewpoint of management (in the light of the 100 issues of the Revista "ROL". Rev Enferm 1986;9(100):3235.

(65) Meléndez i Fernández C. Nursing profession. A past with a future. Rev Enferm 1986;9(100):36-39.

(66) Meléndez y Fernández C. Searching for the lost image. Rev Enferm $1986 ; 9(94): 69-73$.

(67) Suárez Prieto A, Germán Bes $C$. The baker's wife: a nursing predecessor. Rev Enferm 1986;9(94):25-27.

(68) Cuadri Duque MJ. The science and art of childbirth. Historical antecedents to maternal nursing. Rev Enferm 1985;8(84-85):13-16.

(69) Meléndez C. Health and society. Rev Enferm 1985;8(79-80):67-69.

(70) Rodríguez Carballosa L, Delgado García G. Nursing work of Cuban women in the wars for independence from Spain. Rev Cubana Enferm 1985;1(2):103-108.

(71) Barber LH, Skudder S. New Zealanders and the Spanish Civil War. Historical news 1984(48):10-12.

(72) Fernández Gaytán J. Not Available. Revista general de marina 1984;207(Aug-Sep):237-250.

(73) Sanchíz B, de Aguilera N. Berta Sanchíz and Nuria de Aguilera: history of a project. Rev Enferm 1984;7(65-66):36-51.

(74) Alberdi RM. Nursing a woman's profession? Rev Enferm 1983;6(57):2126.

(75) Skudder S, Barber LH. The New Zealand nursing team at the Spanish Civil War. N Z Med J 1983;96(732):397-399.

(76) Díaz F. The influence of Saint Juan de Dios on nursing. Rev Enferm $1982 ; 5(45): 12-14$

(77) Darnés C, Solé A. Influence of religious communities in nursing in Catalonia. Rev Enferm 1981;5(42-43):61-62.

(78) Domínquez Alcón C. History of Catalan nursing. Rev Enferm 1981;5(4243):57-58.

(79) Francisco del Rey C, Fernández de Rota MB. Shaping history, shaping the profession. Nueva Enferm 1980(16):7-8.

(80) Bustamante ME. The 1st nurse in the history of Public Health, Isabel 
Cendala y Gómez. Salud Publica de Mexico 1975;17(3):353-363.

(81) Dieckhoefer K. Spain as the cradle of psychiatry? Nervenarzt 1975;46(12):665-668.

(82) Dieckhoefer K. Lope de Vegas' comedy 'Los locos de Valencia', a view of a 15th century mental asylum. Schweizer Archiv fur Neurologie, Neurochirurgie und Psychiatrie 1975;116(2):343-351.

(83) Kalisch PA. Heroines of '98: female Army nurses in the Spanish-American war. Nurs Res 1975;24(6):411-429.

(84) Martínez Navarro JF. Social factors in the origen of nursing in Spain. Rev Sanid Hig Publica 1975;49(4):343-356.

(85) Jimenez Muñoz JM. Not Available. Asclepio; archivo iberoamericano de historia de la medicina y antropologia medica 1974;26-27:547-553.

(86) Rico-Avello C. Nurses and their history. Caridad Cienc Arte 1971;8(26):3031.

(87) Short history of Spanish hospitals. Caridad Cienc Arte 1970;7(22):10-12.

(88) Usandizaga M. Instruction of male nurses (1624). The first book printed on the technic of care of patients written by a Portuguese in Spanish. Acta Obstet Ginecol Hisp 1970;18(5):309-314.

(89) Anniversary of the Spanish civil war. Cesk Zdrav 1966;14(10):505-510.

Recibido: 17 de agosto 2014; Aceptado 6 de octubre 2014

ISSN 1695-6141

(C) COPYRIGHT Servicio de Publicaciones - Universidad de Murcia 